\title{
El sector solidario como alternativa para el desarrollo social e inclusivo en el posconflicto colombiano
}

\author{
Humberto Serna-Gómez ${ }^{\star}$, Mario Samuel Rodríguez-Barrero ${ }^{* *}$
}

\footnotetext{
EDoctor en Planeación y Política Social, Harvard University. Director del Grupo de Investigación SUMAR, Fundación Universitaria María Cano, Medellín, Colombia

Correo electrónico:

husego@yahoo.com

* Magíster en Dirección de Marketing, Universidad de Viña del Mar. Docente de tiempo completo, Universidad Cooperativa de Colombia, Ibagué, Colombia.

Correo electrónico:

emario.rodriguezb@campusucc.edu.co
}

Recibido: 21 de septiembre del 2015

Aceptado: 6 de noviembre del 2015

Cómo citar este artículo:

\section{Resumen}

Propósito: el artículo busca dar respuesta a la pregunta: ¿cómo puede contribuir el sector de la economía social y solidaria en la inclusión social y laboral para la construcción de la paz? Descripción: a fin de responder a este interrogante se realiza un resumen sobre la evolución del cooperativismo, luego se contextualiza la situación actual del sector de la economía solidaria y, posteriormente, se describe, a manera de ejemplo, la propuesta de investigación bajo el método de caso de la Cooperativa Prosperando. Punto de vista: uno de los factores que ha generado el conflicto es la problemática social de pobreza y desigualdad en la cual se encuentra el país, y la aplicación de los principios de la economía solidaria puede ser una salida viable en el propósito de mejorar la situación de miles de colombianos. Por lo tanto, es importante que se aborden estos temas no solo en el ámbito académico, sino que trasciendan a nivel político, gubernamental $y$, sobre todo, que se hagan realidad. Conclusiones: se concluye que la asociatividad, la inclusión social y la economía civil, como parte del sector de la economía solidaria, son esenciales para la reconciliación y el fortalecimiento de la paz en el posible escenario del posconflicto colombiano.

Palabras clave: asociatividad, economía civil de mercados, economía solidaria, inclusión social. 


\title{
The solidarity sector as an alternative for inclusive social development in the Colombian post-conflict
}

\begin{abstract}
Purpose: the articles aims to answer the question: How can the social and solidarity economy sector contribute to social and labor inclusion for peacebuilding? Description: in order to answer this question, a summary of the evolution of the cooperative movement is presented, then the current situation of the solidarity economy sector is contextualized and, subsequently, the research proposal under the case method of Cooperativa Prosperando is described as an example. Point of view: one of the factors that has given rise to the conflict is the social problem of poverty and inequality in our country, and the application of the solidarity economy principles can be a viable solution to improve the condition of thousands of Colombians. Therefore, it is important that these matters be addressed not only in the academic sphere, but that they transcend politically and governmentally and, above all, that they come true. Conclusions: it is concluded that associations, social inclusion and civil economy, as part of the solidarity economy sector, are essential for reconciliation and peace strengthening in a Colombian post-conflict scenario.
\end{abstract}

Keywords: associations, civil market economy, solidarity economy, social inclusion.

\section{O setor solidário como alternativa para o desenvolvimento social e inclusivo no pós-conflito colombiano}

\section{Resumo}

Propósito: este artigo busca dar resposta à pergunta: como o setor da economia social e solidária pode contribuir na inclusão social e laboral para a construção da paz? Descrição: com o intuito de responder a esse questionamento, realiza-se um resumo sobre a evolução do cooperativismo; em seguida, contextualiza-se a situação atual do setor da economia solidária e, logo, descreve-se, como exemplo, a proposta de pesquisa sob o método de caso da Cooperativa Prosperando. Ponto de vista: um dos fatores que tem gerado o conflito é a problemática social de pobreza e desigualdade na qual a Colômbia se encontra, e a aplicação dos princípios da economia solidária pode ser uma saída viável no propósito de melhorar a situação de milhares de colombianos. Portanto, é importante que sejam abordados esses temas não somente no âmbito acadêmico, mas também que no político, governamental e, principalmente, que se tornem realidade. Conclusões: conclui-se que a associatividade, a inclusão social e a economia civil, como parte do setor da economia solidária, são essenciais para a reconciliação e o fortalecimento da paz no possível cenário do pós-conflito colombiano.

Palavras-chave: associatividade, economia civil de mercados, economia solidária, inclusão social. 


\section{Introducción}

El presente artículo es producto de una ponencia presentada en el I Simposio Nacional Diálogos Empresa y Paz: Perspectivas institucionales, organizado por la Universidad Javeriana en el 2015. La indagación se ubica dentro de la línea de investigación de la economía solidaria, y su importancia radica en el impacto que el sector de la economía social y solidaria puede y debe tener en este momento histórico para el país, en el cual se desarrollan los diálogos de paz y los colombianos tienen la esperanza puesta en la finalización del conflicto y el inicio de una etapa de posconflicto, o por lo menos de posacuerdo.

En relación con el alcance, se puede decir que este tema es de importancia nacional, pues uno de los factores que ha generado el conflicto es la problemática social de pobreza y desigualdad en la cual se encuentra el territorio nacional, y la aplicación de los principios de la economía solidaria puede ser una salida viable para mejorar la situación de miles de colombianos. De esta manera, es importante que se aborden estos temas no solo en el ámbito académico, sino que trasciendan a nivel político, gubernamental $y$, sobre todo, que se hagan realidad.

El propósito del artículo es mostrar a los lectores que el sector solidario es la mejor alternativa para generar desarrollo social, sostenible e inclusivo en Colombia, ante el eventual escenario del posconflicto. Con este fin, se presenta inicialmente una breve historia del cooperativismo mundial; posteriormente, se contextualiza el papel del sector de la economía social y solidaria en Colombia; y, finalmente, se presentan los retos que tienen las empresas de la economía social y solidaria frente al posconflicto, la inclusión social y laboral, y se presenta como un valor agregado un concepto innovador denominado la "Economía Civil de Mercado", el cual podrá ser abordado más ampliamente en una próxima publicación.

\section{Antecedentes del cooperativismo}

Aristóteles, en su Política (1873), conceptúa que el hombre es un ser social. Considera que en todos existe, por naturaleza, la tendencia hacia tal comunidad, lo cual le permite relacionarse con las demás personas. Como consecuencia de esta interacción, se encuentran diferentes maneras de participar en el desarrollo de su comunidad. Algunos lo hacen de manera activa y participativa, otros no. Una de las relaciones que presenta el ser humano es la asociatividad, como una manera de lograr sus propósitos, cuando de modo individual no lo pueden lograr, o cuando varias personas persiguen un mismo objetivo y se dan cuenta como de esta manera será más fácil alcanzarlo. Los procesos asociativos se remontan a los inicios de la vida del hombre, momento en que éste empieza a agruparse con el fin de protegerse de la naturaleza desconocida y del ataque de otros hombres, los cuales buscaban, al igual que ellos, sobrevivir.

La Revolución francesa (1789-1792) marcó un hito en la historia del cooperativismo, gracias a las repercusiones de la caída de instituciones con más de mil años de antigüedad, y dio paso a nuevas forma de gobierno, así como prioridad al individuo, centro de la vida política y social. De esta manera, la sociedad civil se tornó una fuerza comunicadora que debe representar los intereses de la comunidad. No puede faltar el Contrato Social de Rousseau (1836), que sustenta las ideas y doctrinas que apoyan el Estado. Todo esto da como resultado la declaración de los Derechos Humanos.

En el artículo "Historia del Cooperativismo Mundial”, publicado por Ascoop (2011), se precisa que, "en el siglo xix, en Inglaterra, Robert Owen, con sus posiciones, fue considerado el padre de la cooperación inglesa y precursor de las cooperativas de trabajo y producción". Es por esto que Owen siempre ha servido de referente en los procesos productivos y en las teorías salariales, dada la manera como dirigió sus organizaciones, pues permitió que sus empleados participaran de ellas, no solo en la parte laboral, sino también al disfrutar de los beneficios y utilidades de la organización.

Por su parte, Fourier (1772-1837), socialista francés de la primera parte del siglo XIX, propuso la creación de unidades de producción y consumo, las falanges o falansterios basadas en un cooperativismo integral y autosuficiente, así como la libre persecución de lo que llamaba pasiones individuales y su desarrollo, con lo cual construiría un Estado denominado "armonía". Asimismo, pretendió convencer a los capitalistas para que proporcionaran los recursos necesarios en la construcción de falansterios, pero ninguno de ellos aceptó su propuesta.

En 1844, un grupo de obreros ingleses creó una organización cooperativa de carácter legal, con los aportes de sus integrantes. Así fue que el 24 de octubre del mismo año, crearon el primer Almacén Cooperativo en la ciudad de Rochdale, Inglaterra, considerado hoy como el origen de las cooperativas. 
Estaba formado por 28 tejedores desocupados de la fábrica de tejido de Rochdale, quienes habían participado de una huelga, y aportaron este capital como modelo a la nueva sociedad, razón por la cual hoy son conocidos como "Los Pioneros de Rochdale" (Servicio Nacional de Aprendizaje [senA], 1985).

Se puede considerar que estos acontecimientos forman parte de la organización cooperativa que hoy se conoce, ya que ellos fundamentaron su trabajo en principios basados en ideas de solidaridad, dándole formalidad a la organización y un sentido propio, diferente al modelo económico tradicional. Posteriormente, nació la Asociación Cooperativa Internacional (ACI), fundada en Londres, el 19 de agosto de 1895. Desde allí se proclamaron los principios cooperativos como una norma de funcionamiento esencial y doctrinal de las cooperativas. Esta organización es la encargada de abanderar los principios y valores que sustentan la filosofía cooperativista a nivel mundial.

En Colombia, la Confederación de Cooperativas de Colombia (Confecoop) ha sido una de las entidades pioneras en el fomento de los principios cooperativos. Es así como en el XıII Congreso Nacional Cooperativo (2013), el Docente e Investigador Serna, señaló:

La iniciativa empresarial cooperativa en Colombia en sectores diversos, ha estado sujeta a proyectos muy puntuales como salud, educación, servicios funerarios, servicios de vigilancia, industria manufacturera, entre otros, demostrando el potencial que tiene como modelo empresarial en cualquier rama de actividad. El desarrollo de los mencionados subsectores y otros, dependerá del grado de conocimiento y confianza que exista en la población para ver al cooperativismo como esa alternativa (p. 9).

De otro lado, según Martínez (2008), entre los problemas que se encuentran en la sociedad colombiana, están: el bajo nivel de solidaridad, la falta de asociatividad y el individualismo a ultranza que rompe los lazos sociales y debilita las potencialidades para convivir y producir colectivamente. Según este analista, es frecuente que muchas de las iniciativas de asociación se rompan por no saber conciliar los intereses y conflictos, y no fortalecer los lazos de confianza y solidaridad. Bajo este contexto, es indispensable que, desde el Gobierno Nacional, se creen políticas públicas y se ejecuten estrategias con el propósito de fomentar la cultura corporativa y la solidaridad en Colombia.

\section{El sector de la economía social y solidaria en Colombia y su contexto}

\section{La economía social y solidaria en el contexto internacional}

En primer lugar, es importante aclarar que se usa el término economía social y solidaria a fin de caracterizar el sector de la economía solidaria en Colombia. Esto atendiendo a las recomendaciones para la reforma de la Carta Social Andina. Sin embargo, en Colombia, se conoce todavía este sector como el conformado por las cooperativas, fondos de empleados, asociaciones mutuales y las organizaciones solidarias de desarrollo, entre las cuales se encuentran las asociaciones, corporaciones, fundaciones y voluntariado, según lo contemplado en el Decreto 4122 del $2011^{1}$.

En el escenario mundial — caracterizado por el estancamiento de las principales economías-, la solidaridad, la ayuda mutua, la integración y la organización colectiva adquieren vital importancia, ya que puede ser la solución para impulsar el desarrollo social y comunitario y a generar transformación y justicia social. Bajo esta perspectiva, la Asamblea General de las Naciones Unidas proclamó el 2012 como el Año Internacional de las Cooperativas, destacando con ello la contribución que hacen esas entidades al desarrollo de la economía y de la sociedad, especialmente su impacto en la reducción de la pobreza, la creación de empleo y la integración. Considerando también que "las empresas cooperativas ayudan a construir un mundo mejor" según lo definió la onU (Porritelli, 2012, p. 1).

Este desafío propuesto por la onu a las entidades de la economía social y solidaria, tiene tres objetivos: 1) crear mayor conciencia en la sociedad sobre la incidencia de las cooperativas en el desarrollo económico y social; 2) fomentar la constitución y el crecimiento de las organizaciones solidarias para abordar sus necesidades económicas mutuas a través de un modelo de gestión democrático y participativo; 3) incentivar

\footnotetext{
Por el cual se transforma el Departamento Administrativo Nacional de la Economía Solidaria -Dansocial-, en una Unidad Administrativa Especial de Organizaciones Solidarias, se fija su objetivo y estructura. Noviembre 2 del 2011. D.O. N 48241.
} 
a los gobiernos y organismos reguladores a fin de que implementen políticas, leyes y normativas que favorezcan su incremento y desempeño (Porritelli, 2012). En Colombia, este objetivo se puede lograr si se crean leyes desde el Congreso de la República que disminuyan la carga impositiva a este tipo de empresas, ofreciendo créditos blandos a través de bancos de segundo piso como Bancoldex, así como facilitando alianzas público-privadas que faciliten la producción y venta de los productos y servicios que ofrecen estas entidades.

Por su parte, el Secretario General de la Organización de las Naciones Unidas, Ban Ki Moon (citado en Organización de las Naciones Unidas para la Alimentación y la Agricultura, 2012, p. 9), manifestó que "las cooperativas tienen una presencia única e invalorable en el mundo contemporáneo, hacen posible la inclusión social y permiten que prosperen las pequeñas empresas, al tiempo que ayudan a reducir la pobreza y generan empleos decentes". En este pronunciamiento, se evidencia la importancia que tienen las cooperativas en el mundo, pues entre los retos más grandes que tienen hoy en día muchos gobiernos está la reducción de la pobreza a través de la generación de empleos decentes, así como fomentar la inclusión social. En este sentido, el director general de la Organización de las Naciones Unidas para la Alimentación y la Agricultura faO, José Graziano da Silva, afirmó: "la FAO necesita cooperativas y organizaciones de productores fuertes como socios claves en el esfuerzo para eliminar el hambre que sufren cerca de 925 millones de personas y responder a los desafíos del mundo de hoy" (Organización de las Naciones Unidas para la Alimentación y la Agricultura, 2012, p. 9). Según la FAO:

Cerca del $75 \%$ de la población pobre de los países en desarrollo vive en áreas rurales. Una gran parte son pequeños productores que dependen directamente de la agricultura, la pesca, los bosques y el ganado para obtener alimentos e ingresos, pero carecen de acceso a los recursos y oportunidades necesarias para salir de la pobreza extrema (citado por Porritelli, 2012).

De acuerdo con el Informe Ejecutivo de la III Cumbre Social Andina (2012), la economía solidaria se refiere a las unidades productivas fundamentadas en la asociatividad, y las relaciones solidarias que propenden hacia la humanización de la economía de mercado, las cuales buscan bases sociales y comercio justo, dentro de un mundo globalizado. Según el mismo informe, la economía social y solidaria desempeña un papel significativo y creciente en la economía global mediante la provisión de empleo, la protección social y otros beneficios sociales y económicos. En este sentido, es importante resaltar que los pequeños emprendimientos individuales o colectivos en los que se desarrollan iniciativas productivas tendientes a generar encadenamientos de intercambio y de consumo, privilegiando el desarrollo del ser humano dentro de una relación sustentable, hacen parte de la economía social y solidaria.

De acuerdo con lo anterior, se puede afirmar que el sector de la economía solidaria comprende un conjunto de organizaciones creadas por iniciativa privada, las cuales buscan el beneficio colectivo o social, y que tienen como principios comunes la libre adhesión, la democracia, la ausencia de ganancia individual, el desarrollo de la persona natural y la independencia frente al Estado. De otro lado, la Organización Internacional del Trabajo (oIT) reconoce en la Conferencia Internacional del Trabajo (2008) cómo las empresas productivas, rentables y sostenibles, junto con una economía social sólida y un sector público viable, son fundamentales tanto para el desarrollo económico, como para la generación de oportunidades de empleo sostenible. Asimismo, en el Informe Ejecutivo de la III Cumbre Social Andina (2012), se considera que las organizaciones de economía social y solidaria tienen un papel muy importante en la reducción de la pobreza y la promoción de la inclusión social. Por su parte, la Carta Social Andina recomienda a los estados y organizaciones sociales y comunitarias construir un sistema regional de economía solidaria moderna y democrática (Parlamento Andino, 2012).

\section{La economía social y solidaria en Colombia}

Bajo el contexto descrito anteriormente, y si se tienen en cuenta las ventajas que representan las empresas y organizaciones de economía social y solidaria, muchos gobiernos les han dado prioridad a este tipo de organizaciones. Por lo tanto, han creado marcos de política para el desarrollo de estas economías en todo el mundo.

Ateniendo a tales políticas, durante el 2011, se creó en Colombia la Unidad Administrativa Especial de Organizaciones Solidarias, la cual tiene como misión promover, fomentar, fortalecer y desarrollar 
socioempresarialmente las organizaciones solidarias, con el fin de generar progreso en los sectores y regiones del país. Todo esto con una institucionalidad del sector fortalecida y transversal, como parte fundamental de los siguientes cuatro ejes de la nueva organización del Estado: competitividad; política social; inclusión social y reconciliación; eficiencia y buen gobierno. Además, esta unidad asumió las funciones de Dansocial, organismo que estaba encargado de la promoción, el fomento y el fortalecimiento del sector de la economía social y solidaria en el país, conformado por las cooperativas, los fondos de empleados, las mutuales, los grupos de voluntariado, las asociaciones, las corporaciones, las fundaciones y las organizaciones comunales.

Aunado a esto, en Colombia, se cuenta con la Superintendencia de la Economía Solidaria y el Fondo de Garantías para Entidades Cooperativas (Fogacoop), la cual se encarga de asegurar los depósitos para el respaldo a los ahorradores del sector financiero cooperativo. Sin embargo, la actividad cooperativa no debe depender solo de estas entidades, pues este sector es totalmente transversal a la economía y, por ello, se considera que la política pública para el sector no se puede limitar a las instituciones citadas, pues la percepción generalizada es que más allá de las alusiones que hace la Constitución Política, actualmente en Colombia el involucramiento de las cooperativas en programas estratégicos del Gobierno no es claro (Confecoop, 2013b).

Precisamente, respecto a la economía solidaria en Colombia, la Constitución Política de 1991 declara que se "garantiza el derecho de libre asociación para el desarrollo de las distintas actividades que las personas realizan en sociedad...” (Título II, Capítulo I, artículo 38). Más adelante, en el artículo 58, estipula: "El Estado protegerá y promoverá las formas asociativas y solidarias de propiedad"; y reconoce, finalmente: "La empresa como base del desarrollo, tiene una función social que implica obligaciones, el Estado fortalecerá las organizaciones solidarias y estimulará el desarrollo empresarial" (artículo 333, inciso 3).

Por su parte, el director de la Asociación Colombiana de Cooperativas (Ascoop), Carlos Acero Sánchez, según una entrevista concedida en el 2013 al periódico Ecosolidario, considera que es importante crear una política pública incluyente y colectiva para el sector del cooperativismo, así mismo afirmó que el cooperativismo debe integrarse al Estado a través de un nuevo entorno político que esté acompañado de acciones afirmativas, con el fin de permitir la participación y consolidación del movimiento cooperativo. En este sentido, es importante precisar que la política pública que se está adelantando en Colombia para promover el cooperativismo, está contenida en cinco aspectos:

1. Plan Decenal de Desarrollo Cooperativo 2014-2020.

2. Plan Nacional de Desarrollo Nacional.

3. Promoción de un Frente Amplio Parlamentario.

4. Promoción de las Redes de Servicios y Apoyos Cooperativos.

5. Fortalecimiento institucional.

Como ya se precisó, es importante involucrar un mayor número de entidades promotoras del cooperativismo y, sobre todo, se requiere de un amplio compromiso por parte del Gobierno Nacional, los empresarios, el sector público y privado, el sector educativo, el agropecuario y demás organismos, con el propósito de lograr el fortalecimiento del sector de la economía social y solidaria en el país.

\section{Motivos para fortalecer la economía social y solidaria}

Según lo conceptuado por Martínez (2008), la solidaridad y la asociatividad expresadas a través de las organizaciones, sus principios y valores, son un factor fundamental que contribuye a la cohesión social. Según el autor, el papel de estos grupos va a servir de cohesionador en la vida social, pues una sociedad tan dispersa y un Estado que no logra penetrarla profundamente, va a necesitar de un entramado de organizaciones que acerque y cohesione estos individuos, $y$ los aproxime a la vida social. A fin de cumplir esta meta, también se debe fortalecer el emprendimiento solidario y fomentar la asociatividad, ya que el microempresario solidario es quien puede facilitar la construcción de un mejor tejido social en el escenario del posconflicto colombiano, incorporando así a muchas personas a la vida laboral. Sin embargo, esta tarea no se puede hacer con esfuerzos aislados de emprendedores individuales, sino que se debe fomentar la asociatividad como base del capital social.

Según las estadísticas presentadas en el último boletín de prensa del Departamento Administrativo Nacional de Estadística (DANE), con relación a los indicadores de pobreza monetaria y multidimensional en Colombia, durante el 2014 el porcentaje de personas en situación de pobreza fue del $28,5 \%$; es decir, uno de cada tres colombianos es pobre. El informe también reporta como el índice de pobreza extrema 
en la misma vigencia, fue del $8,1 \%$. Una cifra preocupante, si además se tiene en cuenta que el Coeficiente de Gini, asciende a un 0,538, evidenciando así que Colombia es uno de los países con mayor desigualdad en el mundo.

Considerando esta realidad del país, las organizaciones y la sociedad civil deben gestionar iniciativas económicas, comprender adecuadamente los problemas y proponer soluciones, aprendiendo y utilizando nuevas habilidades y conocimientos con la participación de los investigadores, universidades y la academia en general, pues si se fomenta una política clara y una cultura de emprendimiento solidario, se podrá avanzar hacia un proceso de asociación constructiva e intersectorial y hacia la búsqueda de una paz duradera (Parlamento Andino, 2012).

El modelo de economía solidaria ha mostrado ser un importante agente promotor de equidad y desarrollo social, pues, según Martínez (2008), este promueve la formación del capital social, aporta al desarrollo de la democracia participativa, genera y distribuye ingresos, y fomenta la democratización de la riqueza y la propiedad. De esta manera, el sector de la economía solidaria constituye un modelo efectivo de desarrollo socioeconómico solidario e incluyente, y debe ser más protagónico en el mejoramiento de la calidad de vida de las personas a partir de formas asociativas en el país. Según el autor, estos elementos deben contribuir a avanzar hacia la difusión de la cultura de la solidaridad y la asociatividad, y el posicionamiento del sector de la economía solidaria como un modelo integral de desarrollo avanzando hacia una Colombia solidaria y en paz.

\section{Hacia un plan de desarrollo cooperativo}

Según el comunicado de Ascoop (2015), Sector solidario en el Plan Nacional de Desarrollo, la dirigencia cooperativa colombiana, encabezada por Ascoop y Confecoop, presentó una propuesta para que la economía solidaria sea incluida de manera directa y activa en el "Plan Nacional de Desarrollo 2014-2018, Todos por un nuevo país", la cual tuvo una buena acogida por parte del Departamento Nacional de Planeación (DNP). El objetivo de esta propuesta es articular, integrar y promover el sector cooperativo y la economía solidaria en el desarrollo del país durante los próximos cuatro años, enfocándose en los tres pilares claves del plan: paz, equidad y educación (en los cuales el sector solidario ha trabajado históricamente).
La propuesta presentada para incorporar en el Plan de Desarrollo contempla cuatro puntos:

- La valoración de la solidaridad, la asociatividad y la cooperación al interior de las comunidades.

- Cerrar las brechas para promover la asociatividad campesina y la conformación de empresas de carácter cooperativo que fortalezcan la capacidad de los campesinos.

- La inclusión productiva con estrategias innovadoras a fin de impulsar las formas asociativas, las organizaciones de economía solidaria y el modelo cooperativo.

- La educación, contemplada como la formación de docentes y estudiantes en las prácticas y los valores de la asociatividad, la solidaridad y la cooperación, la transparencia, la equidad y la responsabilidad social.

Según lo contemplado en el informe de Confecoop (2013a), Desempeño del Sector Cooperativo Colombiano 2012, la Alianza Cooperativa Internacional (ACI) busca consolidar al movimiento cooperativo como líder reconocido de la sostenibilidad económica, social y medioambiental, de modo que este sea el modelo preferido por la gente y sea el de más rápido crecimiento en las regiones a través de su Visión 2020. Esta estrategia de la ACI, está definida en cinco temas que se ilustran en la figura 1.

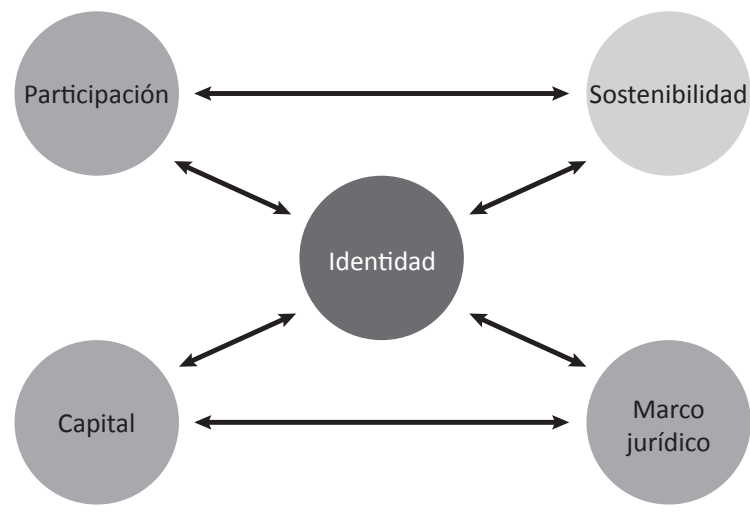

Figura 1. Temas de la Visión 2020. Tomada de Desempeño Sector Cooperativo Colombiano, 2012, por Confeccop, 2013a. Recuperado de: http://confecoop.coop/index.php/noticias? start=4

En concordancia con la Visión 2020, lo que se busca es difundir un modelo empresarial que permita la participación, propenda hacia la sostenibilidad económica, social y ambiental, y tenga como núcleo central e irreductible la identidad cooperativa. Es decir, sus principios y valores. Según el informe de Confecoop (2013a), como en el contexto colombiano 
los diversos temas que resultan relevantes para el desarrollo cooperativo, se han agrupado en los siguientes ejes temáticos:

- Lineamientos de política pública para el sector cooperativo.

- La competitividad como factor de desarrollo de las cooperativas.

- Aporte del cooperativismo al desarrollo económico y social del país.

En el estudio de estos se contempla:

- En el primero, se analizan y evalúan las acciones del Estado a favor de las cooperativas, se incluye el documento Consejo Nacional de Política Económica y Social CONPES para el sector solidario, el rol de los programas públicos, el financiamiento a través de los bancos de segundo piso, y la promoción y el fomento cooperativo, entre otros. A través del desarrollo de estos temas, se busca que el Plan de Desarrollo Cooperativo sea una realidad y contribuya a la transformación social de la economía.

- En el segundo, se busca determinar los factores que incrementan la competitividad del sector cooperativo, incluyendo temas como la doctrina cooperativa, la integración y las redes cooperativas, y la estrategia de comunicaciones, entre otros.

- Finalmente, en el tercer eje, se discute el papel de las cooperativas en el desarrollo social y económico del país, con una mirada desde el desarrollo empresarial y sectorial, el cual históricamente para Colombia se ha construido alrededor de experiencias cooperativas muy puntuales. Se abordarán de manera separada los subsectores financiero, agropecuario, trabajo asociado y otros subsectores; cada uno de ellos identificará las oportunidades que tienen para apoyar la reducción de la desigualdad social y aportar al PIB.

El Estado, por mandato constitucional, debe promover y velar por el desarrollo de las cooperativas; sin embargo, se percibe que algunas agencias gubernamentales desconocen el modelo cooperativo. Además, dada la transversalidad e importancia del cooperativismo, se insiste en que el Ministerio de Educación debería involucrarse con programas educativos sobre el modelo. Por ejemplo, el Ministerio de Agricultura podría impulsar programas a través de cooperativas y el Ministerio de Comercio debería fomentar el emprendimiento y las microempresas cooperativas; en ambos casos, como parte de una sólida política pública.

En relación con el eje de competitividad como factor de desarrollo de las cooperativas, se busca establecer elementos que contribuyan al fortalecimiento de las ventajas competitivas, con base en una gestión diferenciada y soportada en la doctrina cooperativa, pues, según el Informe de Confecoop (2013a), un sentimiento generalizado en el cooperativismo es que el trabajo con las juventudes al interior del cooperativismo colombiano es débil, pese a que organismos como la Alianza Cooperativa Internacional tienen claro que el trabajo con las juventudes es fundamental para formar los dirigentes cooperativos del mañana. En este sentido, se considera que la competitividad del sector se puede mejorar con la asistencia técnica de las universidades y con apoyo del Estado, lo cual se puede ejecutar a través de proyectos de extensión financiados por el Ministerio de Educación, tal como se mencionaba anteriormente.

Es claro que si bien el cooperativismo no busca el lucro, sí tiene un carácter empresarial que debe hacer frente a la economía de mercado, logrando el equilibrio entre fines económicos y fines sociales, lo cual es mucho más fácil si se unen esfuerzos para encontrar una mayor eficiencia de los recursos. De esta manera, si las cooperativas obtienen mejores resultados, como consecuencia de procesos de integración, podrán ampliar su incidencia y visibilidad ante el Estado, los medios de comunicación y la opinión pública, logrando así conseguir mayor beneficio para los asociados.

Por su parte, el eje Aporte del Cooperativismo al Desarrollo Económico y Social del país propone que el sector cooperativo siga presente como una fuerza motora del desarrollo social y económico en Colombia, impactando a cerca del $35,7 \%$ de la población colombiana (Confecoop, 2013a). En el subsector financiero y de crédito, se encuentran aproximadamente la mitad de los asociados a cooperativas en Colombia, y más de la mitad de los activos del sector. Por tanto, se puede catalogar como el subsector líder. El desempeño de este tipo de cooperativas en los últimos años es bastante favorable y ha estado ligado a las situaciones de mercado de crédito y de inversiones para el caso de los seguros. Se puede decir, en términos generales, que se han comportado conforme con las tendencias del mercado financiero.

Igualmente, las cooperativas del sector agropecuario han mostrado un buen desempeño y crecimiento. Sin embargo, problemas tales como la falta de políticas públicas eficientes, el conflicto armado, los cultivos ilícitos, el desplazamiento forzado, la falta de infraestructura vial, la falta de servicios públicos, los bajos niveles de escolaridad, la concentración de las tierras, los bajos niveles de bancarización, entre otros, 
han impedido un desarrollo medianamente cercano al obtenido por las áreas urbanas del país (Confecoop, 2013a).

En relación con las cooperativas de trabajo asociado, se consideró que habían hecho uso indebido del modelo cooperativo, por lo cual el Gobierno Nacional tomó decisiones restrictivas, lo que condujo a una reducción importante de este tipo de cooperativas. Se puede, por tanto, concluir que el sector de la economía social y solidaria en Colombia es diverso; está, por ejemplo, en el campo de la salud, la educación, de los servicios funerarios, de vigilancia, y de la industria manufacturera, entre otros, demostrando así el potencial que tiene como modelo empresarial en cualquier actividad. También es importante resaltar que el desarrollo de los mencionados subsectores y de otros, dependerá del grado de conocimiento y confianza que exista en la población para ver al cooperativismo como una alternativa eficaz.

\section{Los retos de las empresas de la economía social y solidaria frente al posconflicto, la inclusión social y laboral}

La era del posconflicto, como se suele denominar, independiente de si se está de acuerdo o no con esta definición, significa pensar, planear y ejecutar acciones para cuando el conflicto empiece a ser superado. Con esta visión, en el conflicto y en el posconflicto existen múltiples factores y actores que demandan y exigen soluciones integrales y coordinadas, lo cual hace más complejo y desafiante el proceso que permita llegar a una era de posconflicto y a la paz que se anhela.

Con esta visión integral y no parcelada, la pregunta que se busca responder es: ¿cómo pueden contribuir las empresas del sector social y solidario en la construcción de la paz? Y, por otra parte, ¿cuáles son sus retos y desafíos? Como aproximación a una respuesta, los autores vienen trabajando en un proyecto de investigación relacionado con la gestión de las empresas del sector de la economía social, y se ha tomado como ejemplo de estudio de caso la Cooperativa Prosperando de Ibagué, a través de la cual se pretende mostrar cómo una organización asociativa que surge de una crisis del sector de las telecomunicaciones, incorpora laboralmente a un grupo de empleados de Telecom, los mantiene laboralmente activos y asegura el bienestar de sus familiares y su calidad de vida, así como la satisfacción de todos sus afiliados.
A través del caso de la Cooperativa Prosperando, el cual es un ejemplo - como muchos otros- de inclusión social y laboral, se pretenden documentar y difundir aprendizajes de esta experiencia. Además, permitirá contribuir a la discusión, análisis y diseño de estrategias que permitan dimensionar el papel de las empresas de la economía social y solidaria en la construcción de una sociedad más inclusiva, equitativa y justa. Para ello, con este y otros trabajos investigativos, se espera contribuir en una reflexión conceptual sobre los retos y desafíos de este sector como motor de la trasformación social.

Teniendo en cuenta que incluir significa integrar, crear, abrir y diversificar oportunidades dignas, las cuales faciliten el acceso y el mejoramiento de la calidad de vida de poblaciones y personas marginadas sin acceso a los beneficios que otros gozan. Es pasar de una sociedad que excluye a una incluyente. Aquí es donde está el gran reto y la necesidad de que esta estrategia sea integral y sistémica. No es el papel de un solo actor - el Estado o la sociedad civil o colegiada-, es una conjunción de todas las fuerzan que facilitan y hacen posible la inclusión, lo cual significa finalmente una sociedad más justa, equitativa, tolerante y respetuosa de las diferencias y con mayor oportunidad de alcanzar bienestar. Incluir es la construcción de una sociedad que olvida y perdona, generadora de valor compartido para todos.

Para Zamagni (2013), existen diversos modelos económicos, entre los que figuran el neoliberal, propio de los Estados Unidos, cuyo objetivo es incrementar el bien total; y el modelo civil típico de la tradición cultural de Italia y España, el cual pretende incrementar el bien común. Este último es el único que persigue un desarrollo humano integral con tres componentes: el crecimiento, la dimensión sociorrelacional y la espiritual. Este es un nuevo enfoque para la responsabilidad social de las empresas con una marcada responsabilidad ciudadana. Zamagni la denomina economía civil, como aquella que "de una parte, se enlaza con la praxis económica de la floreciente Italia medieval y empalma, por otro lado, con la doctrina social, con principios tales como la solidaridad, la subsidiariedad, y el bien común”. Por otro lado, para Calvo (2013):

Es una propuesta alternativa de economía que incide principalmente en la necesidad de corregir el déficit de fraternidad de las sociedades postindustriales actuales. Esta propuesta trata de mostrar que los problemas económicos, sociales y medioambientales de las economías de mercado y de las sociedades postindustriales 
tienen que ver con la falta de relacionalidad. Fruto de una racionalidad económica fundamentada en el supuesto egoísmo universal de los agentes económicos, este hecho condiciona en gran medida el progreso económico y social al lastrar las posibilidades de satisfacer aquellos bienes relacionales implicados directamente en la autorrealización de las personas (p. 66.).

En conclusión, en la economía civil de mercado, la lógica es la solidaridad y el bien común de sus asociados, no la rentabilidad para unos pocos dueños. En esta definición, encajan entonces un sinnúmero de emprendimientos organizacionales asociativos orientados hacia la generación de valor para sus asociados, tales como juntas de acción comunal, tiendas barriales, famiempresas, fundaciones, fondos de empleados, cajas de compensación, asociaciones de padres de familia, entre otros, y lógicamente las empresas del sector social y solidario. De otro lado, la ACI, en su Visión 2020, define como estrategias para consolidar este modelo organizacional cooperativo las siguientes:

- Participación: las cooperativas son mejores porque permiten la participación de las personas a través de la propiedad, lo que hace que sean inherentemente más atractivas, más productivas, más útiles y más relevantes en el mundo contemporáneo.

- Sostenibilidad: las cooperativas son mejores porque su modelo empresarial crea una mayor sostenibilidad económica, social y medioambiental.

- Identidad: una identidad que se define por los valores y los principios de la cooperación y que se debe comunicar mediante un mensaje claro y poderoso, a fin de garantizar que las cooperativas sean vistas y entendidas por todo el mundo, desde los encargados de determinar las políticas hasta el público en general.

- Marco jurídico: las cooperativas actúan en todas partes dentro de un marco jurídico, el cual desempeña un papel crítico en la viabilidad y la existencia de las mismas.

- Capital: las cooperativas necesitan acceso al capital para establecerse, crecer y florecer.

\section{Los retos y desafíos de las empresas de la economía social y cooperativa en el posconflicto}

Consecuente con este direccionamiento estratégico, tal como lo fórmula la ACI, el sector cooperativo tiene grandes desafíos como actor en una sociedad futura, equitativa e incluyente. Estos son:
- Participación y gobernabilidad: según cifras de la Superintendencia de la Economía Solidaria, "el sector solidario vigilado reporta a 31 de diciembre de 2014, aproximadamente a 6 millones de personas asociadas y genera más de 83 mil empleos" (Supersolidaria, 2015, p. 6).

- Laparticipación: diseñar estrategias para estimular la vinculación y participación de más colombianos en las empresas del sector social y solidario, estableciendo marcos jurídicos que faciliten y apoyen el emprendimiento solidario más allá de los campos de la educación y el crecimiento de empresas asociativas, cooperativas de servicios, agrícolas, mutuales grandes generadoras de oportunidades de inclusión y bienestar. El cooperativismo debería, en sus próximas etapas de desarrollo y en la era del posconflicto, volcarse al campo, su modernización y tecnificación, como la gran oportunidad de inclusión social y laboral. En el Plan Nacional de Desarrollo, esta busca quedar como una prioridad, tal como se afirmó anteriormente.

- La gobernabilidad: la gobernabilidad del sector debería ser igualmente un reto. El carácter democrático de las empresas de la economía social y solidaria se soporta en la asociatividad y en la confianza. Cuando la confianza se vulnera, la asociatividad y la satisfacción de los asociados entran en caos. Modelos de gobernabilidad sólidos, con órganos de gobierno y control claramente establecidos y con credibilidad. Los sistemas trasparentes de rendición de cuentas son absolutamente necesarios para asegurar posicionamiento y competitividad como empresa cooperativa y como sector. El sector, a fin de consolidar su protagonismo, debe recurar su imagen satanizada, como consecuencia de prácticas incorrectas de algunos administradores y gestores.

- La educación cooperativa: consolidar el mensaje cooperativista y definir la identidad de las cooperativas es el nuevo desafío para atraer a la juventud y los grupos sociales que se incorporan a este modelo económico. Regresar a lo básico: la educación cooperativa. Hay que hacer pedagogía del modelo, sus características y beneficios, hay que entrenar y capacitar para la asociatividad, para compartir valor.

El emprendimiento del posconflicto debería ser cooperativo. La cooperativa incluye; su objetivo es generar bienestar, seguridad, calidad de vida. La cooperativa comparte valor para y con todos sus asociados; por tanto, genera bienestar y contribuye a la calidad de vida de sus asociados. 


\section{Las cooperativas como constructoras de sostenibilidad y sustentabilidad}

Ante una inmensa población con necesidades insatisfechas, con muy bajos niveles de participación económica y social, con crisis y ausencia de valores, las empresas cooperativas deberían posicionarse como facilitadoras de sostenibilidad económica y social de sus empresas y sus afiliados, pero sustentables por su compromiso con una responsabilidad social interna y externa, la cual asegure una gestión que genera y comparte valor con sus asociados y sus grupos de referencia.

Conseguir capital fiable para las cooperativas, al mismo tiempo que se garantice la gestión eficiente y trasparente por parte de los miembros, es condición necesaria para asegurar su rol en la construcción de una sociedad en paz. El gobierno nacional y los sectores financiero e industrial, así como la dirigencia política y las entidades de financiamiento internacional, entre otras, deben entender que el sector cooperativo requiere acceso a fuentes de recursos financieros y técnicos que le permitan asegurar su desarrollo y el logro de sus objetivos de inclusión social y laboral. Una estrategia financiera para el sector cooperativo es un elemento fundamental en el propósito de facilitar el éxito de la transición hacia una sociedad en paz. Es indispensable definir políticas y estrategias, de modo que el sector cooperativo pueda acceder a recursos confiables; sin esta opción, será muy poco viable su contribución en la construcción de una sociedad en paz en el corto y, menos aún, en el largo plazo.

Infortunadamente, pareciera que los emprendimientos cooperativos no hacen parte de la agenda del gobierno. No existen proyectos estratégicos de gran impacto para el sector, y hay ausencia de investigación e innovación en el mismo. Alrededor de estos temas girará este proyecto investigativo, el cual se inicia con la Cooperativa Prosperando de Ibagué, departamento del Tolima, y se ampliará hasta llegar a las empresas sociales que integran la economía civil de mercados en Colombia, opción de desarrollo justo e inclusivo generador de valor compartido y calidad de vida.

\section{Conclusiones}

Como se ha argumentado, una buena gestión del sector de la economía social y solidaria puede ser el principal insumo para garantizar una paz social, justicia y equidad duraderas. Por lo tanto, se deben crear programas, políticas y estrategias gubernamentales para fomentar y fortalecer las iniciativas de asociación, las cuales muchas veces se rompen por no saber conciliar los intereses y conflictos, y no fortalecer los lazos de confianza y solidaridad. Debe existir una sensibilización y culturización a nivel nacional sobre la importancia del cooperativismo y la aplicación de estos principios, pues las prácticas desleales del capitalismo, el bajo nivel de solidaridad, la falta de asociatividad y el individualismo han roto los lazos sociales y han debilitado las potencialidades para convivir y producir colectivamente.

Considerando la realidad del país y el posible escenario del posconflicto, las organizaciones y la sociedad civil deben gestionar iniciativas económicas, comprender adecuadamente los problemas y proponer soluciones, aprendiendo y utilizando nuevas habilidades y conocimientos con la participación de la academia en general. Asimismo, retomando lo dicho por Ban Ki Moon (2012), en el sentido de que "las cooperativas tienen una presencia única e invalorable en el mundo contemporáneo, hacen posible la inclusión social y permiten que prosperen las pequeñas empresas, al tiempo que ayudan a reducir la pobreza y generan empleos decentes" (p. 9), se deben fomentar estas formas de asociación, garantizando su sostenibilidad en el tiempo y ofreciendo ventajas e incentivos para que se creen este tipo de empresas.

De acuerdo con lo esbozado en este artículo, se evidencia la necesidad de brindar mayor fortaleza al sector de la economía social y solidaria. Para esto, es necesario el apoyo del Estado, de modo que —desde políticas públicas articuladas desde los distintos ministerios - brinde posibilidades de financiamiento (agricultura familiar, agroecología desde el Ministerio de Agricultura), articulación con universidades para que docentes y alumnos colaboren con emprendimientos de economía social a nivel local, y brindando financiamiento en proyectos de extensión universitaria desde el Ministerio de Educación. En este sentido, se considera que la extensión universitaria debería ser la función que realimenta tanto a la docencia, como a la investigación a partir de las demandas de las comunidades locales. Esto debería ser apoyado por políticas públicas que asignen partidas al financiamiento de proyectos de este tipo, las cuales tengan articulación con organizaciones de economía social en el territorio. Todo este trabajo favorece la cohesión social. 
Las empresas capitalistas en una economía de mercado tendrán un papel muy importante en esta etapa de transición hacia una sociedad que deberá repensar sus valores. La compensación justa, la apertura de nuevas oportunidades laborales y la responsabilidad social interna y externa como una estrategia para compartir valor serán aportes muy importantes a este proceso de transformación. Sin embargo, cuando se trata de inclusión social y laboral, no son las grandes empresas los motores del cambio que se requieren en una etapa de posconflicto; son las pequeñas y medianas empresas y las organizaciones que integran la economía civil las verdaderas inductoras del nuevo modelo de sociedad.

\section{Referencias}

Acero, C. (2014). La participación debe ser un trabajo serio. Ecosolidario. Sección Política pública para el modelo. Bogotá. Recuperado de: http://www.ecosolidario.com.co/eco/ekeko/index.php/component/k2/ item/17 49-\%E2\%80\%98la-participaci\%C3\%B3n-debe-ser-un-trabajo-serio\%E2\%80\%99

Aristóteles. (1873). Política (P. Azcárate, Trad.). Madrid: Biblioteca Filosófica.

Ascoop. (2015). Sector solidario en el Plan Nacional de Desarrollo. Recuperado de: http://www.ascoop.coop/sector-solidario-en-el-plan-nacional-de-desarrollo/

Ascoop. (s. f.). Asociación colombiana de cooperativas. Obtenido de Historia del cooperativismo mundial. Recuperado el 27 de abril de 2015, de: http://www.ascoop. coop/historia-del-cooperativismo-mundial/

Calvo, P. (2013). Fundamentos de la economía civil para el diseño de las organizaciones. Revista Internacional de Organizaciones, 10, 65-84.

Confeccop. (2013a). Desempeño Sector Cooperativo Colombiano, 2012. Recuperado de: http://confecoop.coop/ index.php/noticias?start $=4$

Confeccop. (2013b). xir Congreso Nacional Cooperativo. Sinergia. Para un Plan de Desarrollo Cooperativo. Evento realizado el 8 y 9 de agosto. Cartagena, Colombia.

Constitución Política de Colombia. [Const]. Julio 7 de 1991 (Colombia).

Decreto 4122 del 2011. Por el cual se transforma el Departamento Administrativo Nacional de la Economía Solidaria -DANSOCIAL-, en una Unidad Administrativa
Especial de Organizaciones Solidarias, se fija su objetivo y estructura. Noviembre 2 del 2011. Do No 48241.

Departamento Administrativo Nacional de Estadística. (2015). Pobreza monetaria y multidimensional-2014. Comunicado de prensa. Recuperado de: http://www. dane.gov.co/files/investigaciones/condiciones_vida/ pobreza/cp_pobreza_14.pdf

Martínez, J. (2008). Políticas públicas y economía solidaria en Colombia. II Encuentro Nacional de Educacion. Evento realizado el 6 de agosto, Bogotá, Colombia.

Organización de las Naciones Unidas para la Alimentación y la Agricultura. [FAO]. (2012). Cooperativas campesinas y seguridad alimentaria: Un modelo vigente. Roma: Oficina de Intercambio de Conocimientos, Investigación y Extensión, FAO.

Organización Internacional del Trabajo. [oIT]. (2008). Declaración de la OIT sobre la justicia social para una globalización equitativa, adoptada por la Conferencia Internacional del Trabajo en su nonagésima séptima reunión, 10 de junio del 2008. Ginebra, Suiza.

Parlamento Andino. (2012). Informe Ejecutivo III Cumbre Social Andina. Economía solidaria. Recuperado de http://www.parlamentoandino.org/csa/ documentos-de-trabajo/ informes-ejecutivos/25-economia-solidaria.html

Porritelli, S. (2012). Año Internacional de las Cooperativas. Una alternativa para el cambio. Recuperado de: http://www.centrocultural.coop/blogs/ cooperativismo/2012/03/22/2012-ano-internacional-de-las-cooperativas-una-alternativa-para-el-cambio/

Rousseau, J. J. (1836). El contrato social. Barcelona: Imprenta de los Herederos de Roca.

Servicio Nacional de Aprendizaje. [sena]. (1985). Cartilla N. ${ }^{\circ}$ 1: Generalidades del Cooperativismo. Biblioteca Virtual Luis Angel Arango. Recuperado de http:// www.banrepcultural.org/blaavirtual/ciencias/sena/ cooperativismo/generalidades-del-cooperativismo/ generali1.htm

Serna, H. (2008). Gerencia estratégica. Bogotá: 3R Editores.

Zamagni, E. (2013). La economía civil de mercado es el único modelo que persigue el desarrollo humano integral. Universidad de Comillas. Recuperado de: http://www. eben-spain.org/2013/12/stefano-zamagni-la-economia-civil-de-mercado-es-el-unico-modelo-que-persigue-el-desarrollo-humano-integral/ Consultado el 2 de mayo del 2015. 